\title{
Bridging to Lung Transplant: What Method and for Whom?
}

\author{
Ayyaz A. Ali • Matthew Hartwig • Shu Lin • Robert D. Davis
}

Published online: 30 June 2013

(C) Springer Science+Business Media New York 2013

\begin{abstract}
Worldwide experience and expertise in lung transplant are increasing. For patients with end-stage respiratory disease this therapy greatly improves symptoms, eliminates the need for home oxygen therapy, and restores overall capacity. A major obstacle to increasing its availability as a treatment option is the limited number of donor organs. Patients who develop severe and progressive respiratory failure and are believed to be candidates for this treatment may be eligible for urgent lung transplantation. While waiting for donor organs to become available, methods to support respiratory function are essential to facilitate survival to transplant. Use of extracorporeal support to maintain gas exchange was first described two decades ago and has since developed substantially. We review current techniques available for bridging patients to lung transplant, and the indications and procedures for such support. Recent and historical clinical experience in this field is also discussed.
\end{abstract}

Keywords Lung transplantation - Extracorporeal membrane oxygenation $\cdot$ Respiratory failure $\cdot$ Extracorporeal life support

\section{Introduction}

The number of lung transplants performed worldwide is increasing [1]. However, there is a shortage of organ donors in the face of rising demand. A substantial number of potential donor lungs are assessed as unsuitable for transplant, for reasons including inadequate gas exchange, pulmonary infection, and traumatic injury [2]. Access to organs for patients with severe respiratory disease is therefore limited. The consequences of this situation become most apparent when

A. A. Ali $(\bowtie) \cdot$ M. Hartwig $\cdot$ S. Lin $\cdot$ R. D. Davis

Duke University Medical Center, Durham, NC, USA

e-mail: ayyaz75@gmail.com donor organs are urgently required for patients with an acute and rapid deterioration of respiratory function. If such patients remain suitable for transplant, high levels of support are needed if they are to survive until organs become available. Supporting patients to transplant by use of mechanical devices has been practiced for decades in the field of heart transplantation. There is an increasing realization that similar strategies are needed for patients requiring urgent lung transplantation. This review focuses on describing the rationale, methods, feasibility and previous experience of methods used to bridge patients to lung transplant. Existing techniques and their indications will be discussed and the future of this therapy examined.

\section{Background}

The simplest method of supporting patients who are assessed as suitable for lung transplant but have rapidly declining respiratory function is mechanical ventilation [3]. However, this intervention is often inadequate if the patient's condition deteriorates, requiring consideration of more advanced therapy including extracorporeal membrane oxygenation (ECMO). The earliest use of ECMO as a bridge to lung transplant was reported by Veith et al. in 1975, for a patient with posttraumatic respiratory failure [4]. Over the next two decades sporadic reports of successful ECMO bridging to lung transplant were published, with variable results [4-6]. The Toronto group reported further experience with this technique in the 1980s, and were unable to achieve favorable outcomes for the few patients transplanted after ECMO support [6, 7]. Long-term survival after use of the strategy was first reported by the Hannover group in the early 1990s [5]. ECMO was used for two patients with severe primary graft dysfunction after single lung transplantation. One of these patients was discharged from hospital and was alive at 10-month post-operative follow-up. The other patient died five months after re- 
transplant, after developing airway complications and recurrent viral pulmonary infection. Improvements to materials and component technology have resulted in an increase in ECMO bridging, with several centers recently reporting good initial

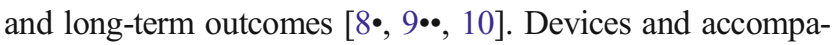
nying procedures that permit ambulation of patients undergoing ECMO have further renewed enthusiasm for the approach $[9 \cdot \bullet$. These procedures may ease patient rehabilitation by avoiding de-conditioning. ECMO support can be instituted via the veno-arterial (VA) or veno-venous route, with cannulation achieved either centrally via the heart and great vessels or via peripheral vessels, for example the femoral artery and vein. The use of the Novalung, a pumpless lung-assist device, for specific situations including refractory hypercarbia has also been reported as a method of successfully supporting patients before lung transplant [11]. Historical experience with ECMO bridging has provided a basis for its more frequent use for patients with fulminant respiratory failure. Indications, purported advantages, and clinical experience regarding ECMO and other bridging strategies will be discussed in this review.

\section{Indications}

The primary indication for bridging to lung transplantation, by use of ECMO or by other means, is rapid, severe deterioration of respiratory function. Severe hypoxemia with a $\mathrm{PaO}_{2}: \mathrm{FIO}_{2}$ ratio lower than $80 \mathrm{~mm} \mathrm{Hg}$ or profound hypercarbia with $\mathrm{PCO}_{2}>80 \mathrm{~mm} \mathrm{Hg}$ can be regarded as objective indications for the use of advanced respiratory support procedures. Hemodynamic and circulatory collapse is another indication, and is commonly observed in patients who have co-existent severe pulmonary hypertension and associated right ventricular dysfunction. Such patients are prone to sudden and severe decompensation. Careful consideration is necessary before instituting ECMO support because the inadequate supply of donor organs, particularly for patients who have a high likelihood of allograft size-mismatching or who are highly sensitized, limits the likelihood of receiving an appropriate organ. Motivated patients with rehabilitation potential and no relevant co-morbid conditions are more likely to achieve good outcomes after pre-operative ECMO support. The cumulative evidence regarding bridging patients to lung transplant by use of extracorporeal devices remains small. There is currently no published single-center study involving more than 50 patients. Consequently, well-established selection criteria for patients suitable for this form of support have not yet been developed. Favorable factors when considering an individual for extracorporeal support include younger patient age and the absence of other dysfunctional organs. Multi-organ dysfunction, sepsis, hemodynamic instability requiring multiple inotropes and vasopressors, prolonged mechanical ventilation, and increased age are unfavorable prognostic factors [12]. Timing of institution of support may also be an important determinant of outcome. Avoiding prolonged intubation and mechanical ventilation via early establishment of ECMO, quickly followed by phased withdrawal of sedation and active rehabilitation, has recently been reported to be associated with excellent outcomes $[9 \bullet \bullet]$.

\section{Extracorporeal Membrane Oxygenation}

Artificial oxygenation and perfusion support to assist cardiac surgery procedures was first developed by John Gibbon in 1953 [13]. Rashkind and colleagues used a bubble oxygenator to support a neonate with severe respiratory failure in 1965 [14]. In the late 1960s Dorson described the use of membrane oxygenators for cardiopulmonary bypass in infants [15]. Widespread use of ECMO to treat severe respiratory illness of neonates was advocated by Bartlett in 1975 [16]. The primary components of an ECMO circuit include a membrane oxygenator, tubing for fluid flow, and a pump for propulsion. Previously, circuits incorporated oxygenators comprised of microporous polyprolene or silicone membranes. More recently polymethylpentene (PMP) membranes have been introduced, which have a number of advantages over previous materials [17]. PMP is a thermoplastic polymer of methylpentene polymer units. Its unique properties include low moisture absorption, high chemical resistance, and very low density, making it very permeable to gases. Oxygenators constructed using PMP membranes enable improved gas exchange, provide lower resistance, reduce plasma leakage, and require less volume for priming. Unlike oxygenators derived from other materials, PMP oxygenators can be heparin coated, reducing the need for systemic heparinization. This, with their material characteristics, improves durability and enables the oxygenator to function for many weeks. PMP oxygenators work in a similar fashion to other hollow-fiber oxygenators: blood flows between the fibers through which oxygenated gas passes, creating a blood-gas interface which enables optimum mixing and gas transfer. In addition to heparin coating of oxygenators, heparin can also be applied as a coat to other components of the circuit, in particular the tubing $[18,19]$. Heparin coating reduces dependence on systemic heparinization and the need for therapeutic anticoagulation. Improvements to materials incorporated into the ECMO circuit are expected to reduce bleeding complications, blood product and platelet consumption, and development of heparin resistance and antibodies. Centrifugal pumps have largely replaced peristaltic roller pumps, reducing spallation, tubing damage, resistance, and blood component trauma [20]. Centrifugal pumps are associated with greatly reduced hemolysis, require less volume for priming, and eliminate the need for a reservoir within the circuit. Advances in design and composition of the ECMO circuit and its components are likely to continue to contribute to their improved safety, durability, and 
feasibility for use in long-term support of patients with cardiac and respiratory failure.

\section{Veno-Venous ECMO}

Veno-venous (VV) ECMO is often regarded as the primary method for supporting patients with severe respiratory failure. Hemodynamic stability is necessary for its institution and maintenance. Usually, a drainage cannula with a diameter of $22 \mathrm{Fr}$ or greater is inserted percutaneously into a femoral vein. Perfusion is achieved via an additional cannula which can be placed in the opposite femoral vein, with its tip beyond the drainage cannula, but is more commonly placed in an internal jugular vein. The infusion cannula is of smaller diameter (17-19 Fr). Flow rate is proportional to patient size, and rates between 3 and $5 \mathrm{~L} \mathrm{~min}^{-1}$ are regarded as necessary for satisfactory oxygenation. Higher flows are believed ideal, improving oxygenation because more blood is drained into the ECMO circuit and thus oxygenated, with a lower proportion passing through the pulmonary circulation and remaining deoxygenated. Oxygenated blood infused into the right atrium mixes with deoxygenated right atrial blood. This mixing reduces the proportion of oxygensaturated hemoglobin in the pulmonary artery. Saturation $>80 \%$ is regarded as acceptable. Ideally the tip of the drainage cannula should be located near the junction of the inferior vena cava and right atrium, and the infusion cannula tip should lie within the superior vena cava above the right atrium. If the cannulae are not separated by a sufficient distance there is insufficient delivery of oxygenated blood into the pulmonary artery. This is the result of infused oxygenated blood being excessively drawn into the drainage cannula rather than entering the right ventricle. A separation of approximately $10 \mathrm{~cm}$ or greater is usually adequate. $\mathrm{VV}$ ECMO support has been simplified by the invention of a bicaval dual lumen catheter by Avalon Laboratories (Rancho Dominguez, CA, USA) [21]. Inflow into the catheter occurs from the cannula tip, which is guided into the inferior vena cava by use of either fluoroscopy or transesophageal echocardiography. Further inflow occurs through fenestrations located more proximally within the cannula, at the junction of the superior vena cava and right atrium. Outflow of oxygenated blood occurs from a location situated between these two drainage points, and is directed toward the tricuspid valve. A small amount of additional oxygenation of blood is often provided by the diseased lungs, improving systemic hemoglobin saturation. For patients whose systemic oxygenation is inadequate, hematocrit greater than $30 \%$ should be maintained to ensure sufficient oxygen delivery. For patients with right ventricular dysfunction or pulmonary hypertension, increasing the sweep gas flow with the objective of achieving a $\mathrm{PCO}_{2}$ in the low $30 \mathrm{~s} \mathrm{~mm}$ $\mathrm{Hg}$ can improve overall hemodynamics and lower pulmonary vascular resistance. Otherwise the sweep gas can be adjusted to target a normal $\mathrm{PCO}_{2}$. Anticoagulation should be instituted, and can be titrated to achieve an activated clotting time (ACT) in the range $150-200 \mathrm{~s}$ or an activated partial thromboplastin time (PTT) in the range 40-60 s. Bleeding complications necessitate a lower target ACT or PTT, and may require avoidance of anti-coagulation. The incidence of adverse events is lower than for veno-arterial ECMO, with reduced bleeding, thrombosis, and cerebrovascular complications [12].

\section{Veno-Arterial ECMO}

Veno-arterial (VA) ECMO is instituted when respiratory failure occurs, either in association with hemodynamic instability or in the context of circulatory failure associated with pulmonary hypertension. For some patients, VV ECMO is tried but persistent hemodynamic instability necessitates conversion to VA ECMO. As for VV, peripheral vessels are preferred for access and are cannulated by use of a percutaneous technique. The femoral vein and artery are the usual sites of cannula insertion. Femoral venous drainage is achieved via a cannula of similar diameter to those used for VV ECMO. Femoral arterial return occurs through a cannula 16-20 Fr in diameter. Retrograde delivery of oxygenated blood via the femoral artery results in an unwanted change to circulatory physiology: deoxygenated blood from the under-ventilated, failing lungs is ejected from the left ventricle into the proximal arterial circulation. Consequently, the oxygen saturation of blood perfusing the lower regions of the body can be substantially greater than that perfusing the heart, head and upper extremities. Augmentation of mechanical ventilation may, via improved pulmonary oxygenation, improve the arterial oxygen saturation of the proximal arterial circulation. An advantage of arterial return via the axillary artery is that this route avoids retrograde delivery of oxygenated blood and the challenges associated with oxygen-poor perfusion to the upper body. Arterial oxygen saturation in the upper extremities must be closely monitored to avoid this potentially dangerous situation. Venous-venoarterial ECMO can be established via hybridization of perfusion by inserting an additional venous inflow cannula into the internal jugular vein. This ensures delivery of oxygenated blood into the pulmonary circulation, avoiding the flow of deoxygenated blood into the left atrium. Another possible complication is distal limb malperfusion. This requires placement of a small (i.e. 14-gauge) catheter distal to the primary arterial cannula. Directly anastomosing a 6-8 $\mathrm{mm}$ hemashield graft enables the axillary artery to be used for inflow. In conjunction with use of the internal jugular vein for venous return, this has the advantage of enabling the patient to be maintained in upright posture and ambulated. After studies on use of awake VV ECMO for spontaneously breathing patients, a similar configuration for VA ECMO has been reported by Olsson et al. and used to successfully bridge four out of five patients to lung transplant [22]. 


\section{Conduct of ECMO}

After commencement of ECMO, mechanical ventilation is adjusted to limit further injury to the lung. Inspired oxygen concentration should be reduced to below $50 \%$. Peak inspiratory pressure should be reduced to low levels $(20-25 \mathrm{~mm}$ $\mathrm{Hg}$ ), the ventilator rate should also be reduced $\left(6-10 \mathrm{~min}^{-1}\right)$, and PEEP should be increased to $10 \mathrm{~mm} \mathrm{Hg}$. Mixed venous oxygen saturation should be in the range $80-85 \%$, and arterial oxygen saturation should ideally be greater than $90 \%$. Negative fluid balance may be beneficial and can be achieved via administration of diuretics or, if necessary, fluid removal via hemofiltration. Antimicrobial use should be extensive and broad to prevent septic complications. In particular, antifungal prophylaxis should be included in the regimen. Temperature should be maintained close to normothermia, although mild hypothermia is often unavoidable. Hemoglobin should be in the range $8-10 \mathrm{~g} \mathrm{dL}^{-1}$. Platelet transfusion is often necessary to address consumption and coagulopathy, particularly when these are associated with bleeding. Anticoagulation is achieved by use of intravenous heparin. For patients receiving ECMO support before transplant, this can be continued for a short time afterwards if there is primary graft dysfunction. Weaning patients from $\mathrm{VV}$ ECMO can be achieved without altering ECMO flow rate, by adjusting gas flow through the circuit. If gas exchange can be maintained at acceptable ventilator settings, ECMO can be discontinued and the patient decannulated. Weaning from VA ECMO requires adequate cardiac and hemodynamic function. As ECMO flow is reduced careful monitoring of blood pressure and central venous pressure, and more invasive measurements, including cardiac output, pulmonary artery pressure, and pulmonary capillary wedge pressure, assist in determining whether hemodynamic function is adequate.

\section{Pumpless Extracorporeal Support: Novolung Interventional Lung Assist (iLA)}

\section{Veno-Arterial}

The Novalung Interventional Lung Assist (iLA) is a membrane ventilator comprising a heparin-coated hollow-fiber PMP membrane (Novalung; Novalung, Heilbronn, Germany) [23]. The device enables oxygen and carbon dioxide gas exchange via simple diffusion. In a similar manner to other membrane oxygenators, the blood-gas interface is formed between the gas within the hollow fibers and the flow of blood around the fibers. The device is designed to operate without a mechanical pump when used in an arterio-venous configuration. Inflow into the iLA is usually via the femoral artery, which is percutaneously cannulated. A portion of the cardiac output, usually 1-2 $\mathrm{L} \mathrm{min}^{-1}$, is diverted through the device for extracorporeal gas exchange. The device is very effective for carbon dioxide removal and has been principally used for treatment of severe hypercapnic respiratory failure [24]. This is a common condition in patients with cystic fibrosis awaiting lung transplant. The Novalung is an alternative to ECMO support for this group of patients; its successful use as a bridge to lung transplant has been reported by Fischer and colleagues at Hannover Medical School. The low resistance of the pumpless arteriovenous mode enables flow of blood through the membrane oxygenator without pump propulsion. Mean arterial blood pressure must be satisfactory to maintain flow through the device. Although very effective at carbon dioxide removal, the small proportion (15-20\%) of total cardiac output oxygenated by the device only minimally increases systemic oxygenation. The device is therefore not appropriate for patients with severe hypoxia $\left(\mathrm{PaO}_{2}: \mathrm{FIO}_{2}\right.$ ratio $\left.<80 \mathrm{~mm} \mathrm{Hg}\right)$. Heparin anticoagulation is necessary, with a target ACT of 150-180 s.

\section{Pulmonary Artery—Left Atrium}

The device has also been used for bridging patients with severe pulmonary arterial hypertension [25]. When this is associated with severe right ventricular dysfunction, both VA and VV ECMO are unable to unload the right ventricle. Consequently there is impairment of blood flow through the pulmonary circulation. Connection of the Novalung between the main pulmonary arteries enables unloading of the right ventricle, with blood passing through the device oxygenated before delivery into the left atrium [26]. Median sternotomy is used to access the heart and great vessels. The pulmonary artery is directly cannulated with a $21-24 \mathrm{Fr}$ catheter, and the left atrial cannula (17-23 Fr) is inserted into the right superior pulmonary vein. This strategy has been revealed to lead to dramatic improvement for patients with decompensated right heart and respiratory failure associated with pulmonary hypertension. Elevated pulmonary artery pressure effectively drives blood flow across the hollow-fiber membrane, removing the need for a pump. After institution of support patients can be extubated and allowed to ambulate and to engage in physiotherapy.

\section{Clinical Experience with Bridge to Lung Transplant}

Toyoda et al. very recently reported their experience with the use of pre-operative ECMO at the University of Pittsburgh [8 ${ }^{\bullet}$. Of 715 patients who underwent lung transplants at their institution between 2005 and 2011, 24 (3.4\%) required pre-operative ECMO support . A further seven patients were placed on ECMO support but died before an organ become available or after a decision that they were no longer suitable for surgical intervention. The mean duration of support was $171 \pm 242 \mathrm{~h}$, with a median support time of $91 \mathrm{~h}$. Clinical outcomes for these 24 patients were compared 
with those for 691 patients who did not need to be bridged to transplant by use of ECMO. The researchers' preferred method of support was veno-venous (VV) perfusion, reserving veno-arterial (VA) ECMO for patients with hemodynamic collapse or severe pulmonary hypertension. Fifteen patients were supported by use of VV ECMO, and the remaining nine received VA ECMO support. Patients who required ECMO were significantly younger than those in the non-ECMO group. ECMO was more commonly required by patients with cystic fibrosis or bronchiectasis, or by those awaiting re-transplant. Bilateral lung transplant via cardiopulmonary bypass was performed for all patients in the preoperative ECMO group. Of the control group just over onethird required cardiopulmonary bypass, with $25 \%$ of patients undergoing single lung transplant. Duration of cardiopulmonary bypass was significantly longer for patients receiving ECMO. Toyoda and colleagues implemented strategies with the objective of maximizing the potential donor pool for patients being supported by use of ECMO. Size mismatch was countered by performing bilateral lobar transplant after back-table lobectomy of allografts for six patients, with all surviving. Over $50 \%$ of patients in the preop ECMO group had severe primary graft dysfunction (PGD) after transplant, with 13 of the 24 patients requiring a period of post-transplant ECMO support. In contrast, only $6 \%$ of patients in the control group required ECMO for severe PGD. Median hospital stay was significantly longer for bridge patients - 47 days compared with 27 days for the control group. Thirty-day survival was comparable-96\% for those with ECMO and $97 \%$ for unsupported patients. Similarly, one and two-year survival were not significantly different for the two groups: one-year ECMO $74 \%$ vs. control $83 \%$, and two-year ECMO $74 \%$ vs. control $74 \%$. Despite a greater incidence of primary graft dysfunction and the frequent need to continue ECMO support after transplant, the researchers emphasize the comparable two-year survival for patients who underwent lung transplant without pre-operative ECMO. In a previous report by the same group, Bermudez et al. reviewed 1,305 patients who underwent lung transplants between 1991 and 2010 [27]. Seventeen of these patients $(1.3 \%)$ received ECMO support before lung transplant. Median duration of support was 3.2 days, and average patient follow-up was 2.3 years. Thirty-day mortality was higher for the ECMO group$19 \%$ in comparison with $7 \%$ for those not requiring ECMO. However, there was no difference in one and three-year survival: one-year ECMO $74 \%$ vs. control $78 \%$, and three-year ECMO $65 \%$ vs. control $62 \%$. Allograft function was also similar, as indicated by measurements of forced vital capacity and forced expiratory volume in one second. A more recent report by this group reported a reduction, compared with their early experience, in 30-day mortality for patients bridged to lung transplant.
This suggests that increased expertise and experience with this technique may improve early outcomes.

Fuehner and colleagues from Hannover report promising results for patients supported with "awake ECMO" [9••]. In this series patients were placed on veno-venous ECMO, using a bi-caval dual lumen catheter for percutaneous veno-venous extracorporeal support. After institution of ECMO patients were extubated, and sedation was discontinued to enable waking and subsequent ambulation. Twenty-six patients bridged to lung transplant by use of awake ECMO were compared with 34 patients supported with conventional mechanical ventilation before transplant. The median duration was nine days for ECMO support and 15 days for ventilation support. Six patients in the ECMO group (23\%) and 10 patients $(29 \%)$ in the mechanical ventilation group died before a donor organ became available. Six-month survival was significantly greater for awake ECMO patients: $80 \%$, in contrast with $50 \%$ for patients who were ventilated. Duration of post-operative ventilation and hospital stay were shorter for those receiving ECMO. Long-term support using this strategy has occasionally been reported, and the potential for ambulation and rehabilitation may substantially improve the patient's prospects of remaining alive until an organ is available. The same group also reports successful VA ECMO for awake and spontaneously breathing patients [23]. Five patients with cardiorespiratory failure associated with pulmonary hypertension were placed on VA ECMO, using only local anesthesia for insertion. This was followed by re-establishment of hemodynamic stability and restoration of gas exchange. Two of the five patients died from bleeding complications. The remaining three patients were supported for 18-35 days before successful lung transplantation. They remained awake and ambulatory and were able to receive physiotherapy. Our experience also confirms the importance of maintaining patients in a state where they remain ambulatory and can participate in physical therapy [28]. Of nine consecutive patients supported between 2007 and 2012, five actively participated in pre-transplant rehabilitation. Post-operatively, these patients required less mechanical ventilation and had reduced ICU and hospital stays. One-year survival after transplant with pre-operative ECMO support was $100 \%$ for these nine patients.

The Hannover group has also revealed that alternative forms of extracorporeal support, for example the Novalung interventional lung assist device, can be used for the same purpose [11]. As mentioned above, this device enables gas transfer across a polymethylpentene membrane via simple diffusion. Fischer et al. used the Novalung to bridge patients with severe hypercapnia which was persistent despite mechanical ventilation. Between 2003 and 2005, 176 lung transplants were performed at Hannover Medical School. Twelve patients awaiting lung transplants had refractory hypercapnia with associated respiratory acidosis. These patients were supported with the Novalung in an attempt to 
bridge them to transplant. The average length of support was $15 \pm 8$ days. Before institution of support, patients were hypoxemic, hypercarbic, and acidotic: $\mathrm{PaO}_{2} 71 \pm 27 \mathrm{~mm}$ $\mathrm{Hg}, \mathrm{pH} 7.12 \pm 0.1$, and $\mathrm{PaCO}_{2} 128 \pm 42 \mathrm{~mm} \mathrm{Hg}$. After cannulation to the Novalung system there was a significant improvement in all of these variables: $\mathrm{PaO}_{2} 83 \pm 17 \mathrm{~mm}$ $\mathrm{Hg}, \mathrm{pH} 7.34 \pm 0.1$ and $\mathrm{PaCO}_{2} 52 \pm 5 \mathrm{~mm} \mathrm{Hg}$. Two patients did not survive to lung transplant, dying from multiple organ failure. Ten patients were successfully transplanted, with eight alive one year later. There were two deaths after transplant, of patients who developed multiple organ insufficiency. Hannover also reports successful bridging to lung transplant for patients with end-stage pulmonary hypertension, using the Novalung to create an oxygenated shunt between the pulmonary artery and left atrium [27]. Four patients were bridged successfully to bilateral lung transplant or heart-lung transplant.

\section{Conclusions}

Clinical techniques for bridging patients with severe respiratory failure to lung transplant are improving. Early experience was sporadic, with disparate results. However, over the past few years several centers around the world have demonstrated that ECMO and other membrane ventilator strategies can be used to stabilize patients while they await a donor organ. Continual improvements to ECMO technology are rendering this therapy safer and more feasible even for long-term support. Reduced need for anticoagulation, diminished trauma to blood components and improvement to the composition of hollow-fiber oxygenators are some of the features which increase the clinical suitability of ECMO as a bridge to transplant. Accumulating experience and success with the use of ECMO for patients who are spontaneously breathing and are able to eat and mobilize is a major achievement in the more widespread acceptance of mechanical support as a bridge to lung transplant. Focused efforts to develop improved artificial lung technology promise to deliver more compact and patient-friendly devices, which may increase the potential for ambulation during support and possibly even enable discharge from hospital. Although patients who require extraordinary support are at increased risk of morbidity and mortality after transplant, outcomes have greatly improved and are comparable with those achieved for conventional lung transplant patients. The use of ambulatory ECMO approaches seems to mitigate some of the myopathic and multi-organ system dysfunction complications common with use of mechanical ventilation or with non-ambulatory ECMO. This has substantially reduced the resources needed after transplant. Understanding of the appropriate timing for instituting ECMO and of how to select the correct support configuration will be essential in ensuring that the excellent results reported by centers with current experience can be reproduced at all institutions with a lung transplant program. Continued refinement and improvement of ECMO and associated therapy promises improved prospects for patients with end-stage lung disease who are awaiting transplant.

\section{Compliance with Ethics Guidelines}

Conflict of Interest Ayyaz A. Ali declares that he has no conflict of interest.

Matthew Hartwig declares that he has no conflict of interest.

Shu Lin declares that he has no conflict of interest.

Robert D. Davis declares that he has no conflict of interest.

Human and Animal Rights and Informed Consent This article does not contain any studies with human or animal subjects performed by any of the authors.

\section{References}

Papers of particular interest, published recently, have been highlighted as:

- Of importance

•- Of major importance

1. Mahida RY, Wiscombe S, Fisher AJ. Current status of lung transplantation. Chron Respir Dis. 2012;9(2):131-45.

2. Fisher AJ, Donnelly SC, Pritchard G, Dark JH, Corris PA. Objective assessment of criteria for selection of donor lungs suitable for transplantation. Thorax. 2004;59(5):434-7.

3. O'Brien G, Criner GJ. Mechanical ventilation as a bridge to lung transplantation. J Heart Lung Transplant. 1999;18(3):255-65.

4. Veith FJ. Lung transplantation. Transplant Proc. 1977;9:203-8.

5. Jurmann MJ, Haverich A, Demertzis S, Schaefers HJ, Wagner TO, Borst HG. Extracorporeal membrane oxygenation as a bridge to lung transplantation. Eur J Cardiothorac Surg. 1991;5:94-7.

6. The Toronto Lung Transplant Group. Sequential bilateral lung transplantation for paraquat poisoning. A case report. J Thorac Cardiovasc Surg. 1985;89:734.

7. Nelems JM, Rebuck AS, Cooper JD, Goldberg M, Halloran PF, Vallend H. Human lung transplantation. Chest. 1980;78(4):569-73.

8. - Toyoda Y, Bhama JK, Shigemura N, Zaldonis D, Pilewski J, Crespo M, et al. Efficacy of extracorporeal membrane oxygenation as a bridge to lung transplantation. J Thorac Cardiovasc Surg. 2013;145(4):1065-71. This study describes one of the largest published experiences of use of ECMO as a bridge to lung transplantation.

9. •- Fuehner T, Kuehn C, Hadem K, Wiesner O, Gottlieb J, Tudorache I, et al. Extracorporeal membrane oxygenation in awake patients as bridge to lung transplantation. Am J Respir Crit Care Med. 2012;185(7):763-8. This study demonstrates that ECMO as a bridge to lung transplantation for awake, extubated, and ambulatory patients is associated with a significant survival advantage after transplantation, in comparison with patients who were supported with mechanical ventilation before transplant.

10. Hammainen P, Schersten H, Lemstrom K, Riise GC, Kukkonen S, Sward K, et al. Usefulness of extracorporeal membrane oxygenation as a bridge to lung transplantation: a descriptive study. J Heart Lung Transplant. 2011;30(1):103-7. 
11. Fischer S, Simon AR, Welte T, Hoeper MM, Meyer A, Tessman R, et al. Bridge to lung transplantation with the novel pumpless interventional lung assist device Novalung. J Thorac Cardiovasc Surg. 2006;131(3):719-23.

12. Fischer S, Bohn D, Rycus P, Pierre AF, de Perrot M, Waddell TK, et al. Extracorporeal membrane oxygenation for primary graft dysfunction after lung transplantation: analysis of the Extracorporeal Life Support Organization (ELSO) registry. J Heart Lung Transplant. 2007;26(5):472-7.

13. Miller BJ, Gibbon Jr JH, Greco VF, Smith BA, Cohn CH, Allbritten Jr FF. The production and repair of interatrial septal defects under direct vision with the assistance of an extracorporeal pump-oxygenator circuit. J Thorac Surg. 1953;26(6):598-616.

14. Rashkind WJ, Freeman A, Klein D, Toft RW. Evaluation of a disposable plastic, low volume, pumpless oxygenator as a lung substitute. J Pediatr. 1965;66:94-102.

15. Dorson Jr W, Baker E, Cohen ML, Meyer B, Molthan M, Trump $\mathrm{D}$, et al. A perfusion system for infants. Trans Am Soc Artif Intern Organs. 1969; 15:155-60.

16. Bartlett RH, Gazzaniga AB, Fong SW, Jefferies MR, Roohk HV, Haiduc N. Extracorporeal membrane oxygenator support for cardiopulmonary failure. Experience in 28 cases. J Thorac Cardiovasc Surg. 1977;73(3):375-86.

17. Toomasian JM, Schreiner RJ, Meyer DE, Schmidt ME, Hagan SE, Griffith GW, et al. A polymethylpentene fiber gas exchanger for long-term extracorporeal life support. ASAIO J. 2005;51(4):390-7.

18. Von Seggesser LK. Heparin-bonded surfaces in extracorporeal membrane oxygenation for cardiac support. Ann Thorac Surg. 1996;61(1):330-5.

19. Nojiri C, Hagiwara K, Yokoyama K, Kurbayashi E, Hidaka K, Ishida $\mathrm{N}$, et al. Evaluation of a new heparin bonding process in prolonged extracorporeal membrane oxygenation. ASAIO J. 1995;41(3):561-7.
20. Green TP, Kriesmer P, Steinhorn RH, Payne NR, Irmiter RJ, Meyer CL. Comparison of pressure-volume flow relationships in centrifugal and roller pump extracorporeal membrane oxygenation systems for neonates. ASAIO Trans. 1991;37(4):572-6.

21. Bermudez CA, Rocha RV, Sappington PL, Toyoda Y, Murray HN, Boujoukos AJ. Initial experience with single cannulation for venovenous extracorporeal oxygenation in adults. Ann Thorac Surg. 2010;90(3):991-5.

22. Olsson KM, Simon A, Strueber M, Hadem J, Wiesner O, Gottlieb $\mathrm{J}$, et al. Extracorporeal membrane oxygenation in nonintubated patients as bridge to lung transplantation. Am J Transplant. 2010;10(9):2173-8.

23. Jegger D, Revelly JP, Horisberger J, Mallabiabarrena I, Seigneul I, Jacchertz M, et al. Ex vivo evaluation of a new extracorporeal lung assist device: Novalung membrane oxygenator. Int J Artif Organs. 2005;28(10):985-99.

24. Ricci D, Boffini M, Del Sorbo L, El Qarra S, Comoglio C, Ribezzo M, et al. The use of $\mathrm{CO} 2$ removal devices in patients awaiting lung transplantation: an initial experience. Transplant Proc. 2010;42(4):1255-8.

25. Camboni D, Philipp A, Arlt M, Pfeiffer M, Hiker M, Schmid C. First experience with a paracorporeal artificial lung in humans. ASAIO J. 2009;55(3):304-6.

26. Strueber M, Hoeper MM, Fischer S, Cypel M, Warnecke G, Gottlieb J, et al. Bridge to thoracic organ transplantation in patients with pulmonary arterial hypertension using a pumpless lung assist device. Am J Transplant. 2009;9(4):853-7.

27. Bermudez CA, Rocha RV, Zaldonis D, Bhama JK, Crespo MM, Shigemura N, et al. Extracorporeal membrane oxygenation as a bridge to lung transplant: midterm outcomes. Ann Thorac Surg. 2011;92(4):1226-31

28. Rehder KJ, Turner DA, Hartwig MG, Williford WL, Bonadonna D, Walczak RJ Jr, et al. Respir Care. 2012 [Epub ahead of print] 